\title{
Morphological characteristic of intracerebral hemorrhage in rats and correlation of its volume with results of behavioral tests
}

\author{
Kyrylo M. Zolotko ${ }^{1}$, Oleksandr M. Sukach ${ }^{2,3}$, Antonina M. Kompaniiets ${ }^{1}$, Kateryna S. Liubomudrova ${ }^{4}$
}

${ }^{1}$ Department of Cryoprotectants, Institute for Problems of Cryobiology and Cryomedicine of the National Academy of Sciences of Ukraine, Kharkiv, Ukraine

2 Department of Cryobiochemistry, Institute for Problems of Cryobiology and Cryomedicine of the National Academy of Sciences of Ukraine, Kharkiv, Ukraine

${ }^{3}$ Ya.R. Sinelnikov Department of Human Anatomy and Physiology, H.S. Skovoroda Kharkiv National Pedagogical University, Kharkiv, Ukraine

${ }^{4}$ Department of the Clinical Anatomy and Operative Surgery, Kharkiv National Medical University, Kharkiv, Ukraine

Received: 27 December 2020 Accepted: 09 February 2021

Address for correspondence: Kyrylo M. Zolotko, Department of Cryoprotectants, Institute for Problems of Cryobiology and Cryomedicine, 23 Pereiaslavska str., Kharkiv, 61015, Ukraine, e-mail: zolotkokm@gmail.com
The intracerebral hemorrhage is associated with severe complications and high mortality. Currently there are no effective methods of treatment of this disease while the standard collagenase model of intracerebral hemorrhage is not described sufficiently.

Objective. To analyze morphological characteristics of the collagenase model of intracerebral hemorrhage, and develop the regression formula predicting the hemorrhage volume based on the results of behavioral tests.

Materials and methods. The experiments were carried out on 7 white male rats weighing $250-400 \mathrm{~g}$ aged $11-13$ months. All animals underwent surgery to simulate intracerebral hemorrhage. Rats were anesthetized and then, stereotactically, using a needle with a diameter of $0.47 \mathrm{~mm}, 0.2$ units of collagenase type IV were slowly injected into the left striatum. The day after the intracerebral hemorrhage, functional disabilities developed in rats were studied using beam walking test, neurological score test and adhesive removal test. Immediately after performing behavioral tests, the rats were sacrificed by decapitation. After the brain formalin fixation, serial sections on a vibromicrotome of $200 \mu \mathrm{m}$ thick each in the anteroposterior direction with following morphological examination were made.

Results. It was revealed that the collagenase model of intracerebral hemorrhage is associated with a large variability of the hemorrhage volume. It also had an irregular rugged shape and marks of repeated diapedetic hemorrhages of about $0.6 \mathrm{~mm}$ depth. The center of intracerebral hemorrhage along the anteroposterior axis was in average $0.5 \mathrm{~mm}$ posterior of the actual site of collagenase injection.

The combined use of the neurological score test, the beam walking test and the adhesive removal test in the collagenase model can help estimate the probable intracerebral hemorrhage volume on the 1st day using the regression formula.

Conclusions. Technical details identified in our study can help researchers in planning and conduction of correct experiments related to intracerebral hemorrhage.

Key words: intracerebral hemorrhage; rats; behavioral tests; morphometry

\section{Introduction}

Today intrace rebral hemorrhage (ICH) is the second most common cause of strokes, it is accompanied by severe complications and has high mortality rate. The case fatality rate of $\mathrm{ICH}$ is $54 \%$ at 1 year, and only $12 \%$ to $39 \%$ of survivors can achieve long-term functional independence. [1] Effective treatments for $\mathrm{ICH}$ have not been developed yet $[2,3]$. In this regard there is a need to find new methods of treatment. Therefore, the development and detailed description of effective $\mathrm{ICH}$ models in laboratory animals is an important task for researchers. For animal studies, two methods of $\mathrm{ICH}$ modeling are most often used: the injection of either autologous blood or collagenase into the striatum [4]. The second method reflects the real clinical picture of the disease in humans better, as it is associated with ongoing hemorrhage from the blood vessels of the brain, rather than one-time injection of blood [4]. It should be mentioned that the way of the distribution of hemorrhage in the collagenase model can differ significantly from that with the injection of autologous blood, because with the injection of collagenase we can expect diapedetic hemorrhage of a difficultly predicted shape and amount, which may be due to individual features of the vessels location and the variability of places of injection, while autologous blood is always injected in a given volume into a specific area of the brain [4].

Despite the advantages of using collagenase for $\mathrm{ICH}$ modeling, extensional characteristics of hemorrhage in this model have not been described yet. At the same time, there are no cheap and effective ways of preliminarily estimation the volume of hemorrhage.

The aim of our research was therefore to study variations of the amount and extensional distribution 
of hemorrhage with a collagenase model of $\mathrm{ICH}$, as well as developing a method for preliminary estimation of hemorrhage in rats based on behavioral tests. The goal was also to simplify and optimize the selection of experimental animals with a developed ICH model.

The objective of the work was to study the amount and extensional distribution of intracerebral hemorrhage (ICH) caused by the injection of collagenase into the striatum, as well as the development of a regression model allowing the estimation of the size of $\mathrm{ICH}$ in rats according to the results of behavioral tests.

\section{Materials and methods}

The study was carried out in accordance with the principles of bioethics, regulated by the European Convention for the Protection of Vertebrate Animals used for Experimental and other Scientific Purposes (1986), Directive 2010/63/EU "On the Protection of Animals Used for Scientific Purposes" (2010), the Law of Ukraine No. 3447-IV "On the Protection of Animals from Cruelty" (2006) and approved by the Ethics and Bioethics Commission of Institute for Problems of Cryobiology and Cryomedicine of the NAS of Ukraine (Meeting Minutes No 5 of 08 December 2020).

\section{Intracerebral Hemorrhage modeling}

The experiments were carried out on 7 white outbred male rats weighing $250-400 \mathrm{~g}$, aged $11-13$ months. All animals underwent surgery to simulate ICH [5]. To perform the surgery, the rats were anesthetized by intraperitoneal injection of $15 \mathrm{mg} / \mathrm{kg}$ propofol (Daewon Pharmaceutical Co. Ltd. Korea/Switzerland) and $5 \mathrm{mg} / \mathrm{kg}$ xylazine (Biowet Puławy Sp., Poland). After that stereotactically (coordinates from bregma: anteroposterior: $0.2 \mathrm{~mm}$, mediolateral on the left side: $3.0 \mathrm{~mm}$ ) [6], using a needle with a diameter of $0.47 \mathrm{~mm}$, 0.2 units of type IV collagenase ("Sigma-Aldrich", USA) were slowly introduced in the striatum to a depth of 6.0 $\mathrm{mm}$ in $1 \mu \mathrm{l}$ of physiological saline. 5 minutes after the injection, the needle was removed, and the wound was sutured. The rats were allowed to recover from surgery in a warm environment for 24 hours before the beginning of the series of behavioral tests.

\section{Behavioral tests}

Beam Walking test (BWt) [7] was performed to study the coordination of movements and the ability to maintain balance. The rats crossed a cylindrical wooden beam $70 \mathrm{~cm}$ long and $4 \mathrm{~cm}$ in diameter which was placed $100 \mathrm{~cm}$ above the floor and connected to a dark box where the food was placed. The performance was video recorded. Training was repeated three times. The results were graded using a modified scale [8]: score 0 , the rat passes through the beam without foot slips; score 1, the rat traverses while grasping the lateral side of the beam; score 2 , the rat slips quite often, but passes through the beam; score 3, the animal takes a long time to pass the beam due to difficulty walking; score 4 , the rat is unable to pass the beam; score 5 , the rat cannot move on the beam; score 6 , the animal cannot hold on the beam for more than $10 \mathrm{~s}$.

Adhesive Removal test (ARt) [7] was performed to study tactile sensory dysfunction. Two adhesive tapes of the same size (11 cm Urgosyval ${ }^{\circledR}$ Laboratoires Urgo, France) were applied with equal pressure on each palm of the animal forepaw. The effectiveness of the tactile response was graded by the time which the animals needed to remove the adhesive tape., The animals were trained thrice before the surgery. The maximum measurement duration was $180 \mathrm{~s}$. In most cases, rats needed less than $80 \mathrm{~s}$ to remove the adhesive tape, therefore, for the effectiveness of interpretation, the time for removing the adhesive tape was transferred to a 5-point scale: 4 points - more than $180 \mathrm{~s}$ (did not remove the tape until the end of the test); 3 points from 80 to $180 \mathrm{~s} ; 2$ points $-40-80 \mathrm{~s} ; 1$ point $-20-40$ s; 0 points - less than $20 \mathrm{~s}$.

During the Neurological Score test (NSt) [7], contralateral sensorymotor functions were examined according to 8 parameters, each of them was estimated as 1 point (Table 1 ).

To study the grasping reflex, a $2 \mathrm{~mm}$ steel rod was used. To evaluate the righting reflex, the animal was held in lumbosacral region, while the body was turned in the ipsilateral region, the head and neck were turned in the opposite direction, which led to straightening. To assess placing reactions, the rat was placed on the edge of a table so that the contralateral limbs were hanging

Table 1. Scores for assesment of sensorymotor functions

\begin{tabular}{|l|l|c|c|}
\hline \multicolumn{2}{|c|}{ Functions } & Normal score & Deficiency \\
\hline \multirow{2}{*}{ Grasping reflex } & Forelimb & 1 & 0 \\
\cline { 2 - 4 } & Hindlimb & 1 & 0 \\
\hline \multirow{2}{*}{ Righting reflex } & 1 & 0 \\
\hline Placing reactions & Forelimb hanging down & 1 & 0 \\
\cline { 2 - 4 } & Back paw hanging down & 1 & 0 \\
\cline { 2 - 4 } & Visual support reaction & 1 & 0 \\
\hline \multirow{3}{*}{ Tail suspension } & Forelimb flexion & 1 (absent) & 0 (present) \\
\cline { 2 - 4 } & Torso twisting & 1 (absent) & 0 (present) \\
\hline \multirow{2}{*}{ Total neurological score (the sum of scores) } & 8 & 0 \\
\hline
\end{tabular}


down. The rats automatically returned both paws onto the edge of the table. To study the reaction of visual support, the rat was held by the tail and slowly brought to the edge of the table. Normally, the rat reached the table with both paws, including the contralateral one. When being suspended, the rats were also examined for abnormal postures: contralateral forelimb flexion and torso twisting.

All tests were carried out from 10 am to $1 \mathrm{pm}$.

\section{Morphology}

Immediately after performing behavioral tests, rats were anesthetized by $15 \mathrm{mg} / \mathrm{kg}$ propofol (Daewon Pharmaceutical Co. Ltd. Korea/Switzerland) and 5 $\mathrm{mg} / \mathrm{kg}$ xylazine (Biowet Puławy Sp., Poland) and than decapitated. The animals' brains were extracted and fixed in $10 \%$ neutral phosphate-buffered formalin (3.7-4.0\% formaldehyde) (O.L.KAR.), pH 7.0, at room temperature for 30-32 hours. The fixative volume exceeded the tissue volume by more than 10 times. After the formalin fixation, the specimens were glued to a plate of the vibromicrotome (Leica VT1000S, Germany) and a serial of frontal sections were made of $200 \mu \mathrm{m}$ thick each in the anteroposterior direction in the amount corresponding to the amount of hemorrhage. The sections were not stained, since the peripheral hemorrhages were contrasted with the pigment hemosiderin, which came from the destruction of red blood cells by migrating macrophages [9]. Microscopic analysis and microphotography of sections were made with LSM 510 META microscope (Karl Ztiss, Germany). The area and length of the objects were measured using Zeiss LSM Image Examiner program. The following formula [10] was used to calculate the volume of $\mathrm{ICH}$ in Ms Excel:

$$
\sum_{i=1}^{n}=\mathrm{S} i \times \mathrm{t}(1)
$$

where $\mathrm{S}$ is the section area $\mathrm{i}$, and $\mathrm{t}$ is section cut thickness $(200 \mu \mathrm{m})$.

To make possible the 2 d-illustration of $\mathrm{ICH}$, which is irregular 3-dimensional object, it was necessary to calculate diameters from the areas of the ICH sections assuming (for this purpose only!) that all sections are round in shape

$$
D=2 \sqrt{\frac{s}{\pi}}(2)
$$

where $D$ is the diameter of the circle, $S$ is the section area.

This was necessary to display anterior-posterior volume distribution as if it was a longitudinal section through a rounded hemorrhage.

To assess the contours of hemorrhage, the shape factor was calculated by the formula [10]:
$\mathrm{SF}=4 \pi \times \mathrm{S} / \mathrm{P}^{2}(3)$

where SF is the Shape Factor, $\mathrm{S}$ is the section area, $P$ is the perimeter.

When SF $=1$ - the object has a round shape; the minimum value corresponds to the most rugged edge.

\section{Data analysis}

To rate the correlation between quantitative (can be counted) and qualitative (presence or absence of a sign) data, the point biserial correlation coefficient $(\mathrm{Rpb})$ was used, i.g. between area of the hemorrhage and the probability of detecting 'clear zone' in the center of ICH [11]. The Pearsons linear or curvilinear correlations were used to estimate the dependency between two quantitative (measurable) signs [11,12], i.g. between Neurological Score Test and volume of $\mathrm{ICH}$. Linear regression was used to predict ICH volume by INE coefficient. In all cases, the significance level of $a=0.05$ was accepted. The median (1st quartile; 3rd quartile) was used as the average value and description of distribution in case of small samples and/ or non-parametric distribution.

\section{Results and discussion}

Morphological analysis of the sections showed that the injection of collagenase destroyed the blood vessels of the brain and caused hemorrhage, the amount as well as the anteroposterior and transverse dimensions of which are shown in Table 2.

When analyzing microspecimens, traces of needle insertion were found (Fig. 1), the localization of which was determined using a stereotactic atlas [6] and corresponded to the following coordinates from bregma: mediolateral: $3.0(3.0 ; 3.2) \mathrm{mm}$, and anteroposterior: $1.2(1.0 ; 1.5) \mathrm{mm}$ (see Table 3). In the sections through the central part of the $\mathrm{ICH}$, a moderate elongation of the hemorrhage was detected in the dorsoventral direction, which repeated the way of inserting the needle to simulate ICH.

Since the estimated anteroposterior (AP) position of inserted needle was $0.2 \mathrm{~mm}$, it can be concluded that the needle is systematically deflected anteriorly by an average of $1.0 \mathrm{~mm}$, possibly due to the needle skew, directed by the sharp end to the anterior, and the hole to the posterior. The detected deflection was largely compensated by a characteristic feature of the hemorrhage's distribution, mainly in the posterior direction from the place of injection (Fig. 2). This can be explained by turning the needle hole back, or/and by peculiarities of histology and microanatomy of local vessels, as well as the fact that more soft tissues are located posteriorly to the hematoma, which, potentially, can give more space when the blood is forced out under

Table 2. Morphological characteristics of hemorrhages

\begin{tabular}{|l|r|r|r|r|r|r|r|r|}
\hline & $\mathbf{n}$ & $\mathbf{m i n}-\mathbf{m a x}$ & $\mathbf{Q}_{\mathbf{2 5}}$ & $\mathbf{Q}_{\mathbf{5 0}} \mathbf{( M e )}$ & $\mathbf{Q}_{\mathbf{7 5}}$ & \multicolumn{1}{c|}{$\mathbf{S k}$} & \multicolumn{1}{c|}{$\mathbf{K}$} & \multicolumn{1}{c|}{$\mathbf{A M}$} \\
\hline Amount of $\mathrm{ICH}, \mathrm{mm}^{3}$ & 7 & $2.8-27.3$ & 7.6 & 17.3 & 23.8 & -0.2 & -1.9 & 15.7 \\
\hline Anteroposterior size of $\mathrm{ICH}, \mathrm{mm}$ & 7 & $1.8-4.4$ & 3.0 & 3.1 & 3.9 & -0.5 & 0.4 & 3.3 \\
\hline Transverse size of $\mathrm{ICH}, \mathrm{mm}$ & 7 & $1.8-3.8$ & 2.3 & 3.5 & 3.7 & -0.7 & -1.7 & 3.0 \\
\hline
\end{tabular}

Where: min-max is the minimum and maximum value, $\mathrm{Q}_{25}, \mathrm{Q}_{5}$ and $\mathrm{Q}_{75}$ are the $1 \mathrm{st}$, 2 nd, and 3rd quartiles respectively; $\mathrm{Me}$ is the median; Sk - skewness; $\mathrm{K}$ - kurtosis; $\mathrm{AM}$ - arithmetic mean; ICH - intracerebral hemorrhage. 
the pressure of the forming hematoma with subsequent micro-ruptures of tissues. As a result, the central part of the ICH in the AP direction was less deflected from the preplanned needle insertion place $(0.4(0.3 ; 0.6) \mathrm{mm})$ than might be expected in this case $(1.0(0.8 ; 1.3) \mathrm{mm})$ (Tab. 3; Fig. 2).

Analyzing the serial sections of the brain, it was discovered that the $\mathrm{ICH}$ border has an irregular shape $(\mathrm{SF}=0.32(0.20 ; 0.50))$, which indicates uneven distribution of the spilled blood in the brain tissue (Fig. 1; 3; Table 3).

With the hemorrhage area having more than 4 $\mathrm{mm}^{2}$, a noticeably less hemosiderin-stained "clear zone"
(CZ) was distinguished in the center, which was always surrounded by an intense staining band of $0.56(0.49$; 0.59) $\mathrm{mm}$ thick (Fig. 1; 3). The ICH area statistically significantly positively correlated with the probability of detecting $\mathrm{CZ}$ in the center of $\mathrm{ICH}(\mathrm{Rpb}=0.84 ; p=0.001)$

(Fig. 4; Table 4). Apparently, $C Z$ is an ischemic tissue with damaged vessels that are a result of collagenase injection, while the "marginal zone" (MZ) corresponds to the areas which border with uninjured vessels. It results in multiple microbleeds [4,13]. A large amount of hemosiderin can be explained by the activity of macrophages which phagocytose red blood cells and as a result hemosiderin is formed from hemoglobin [9].
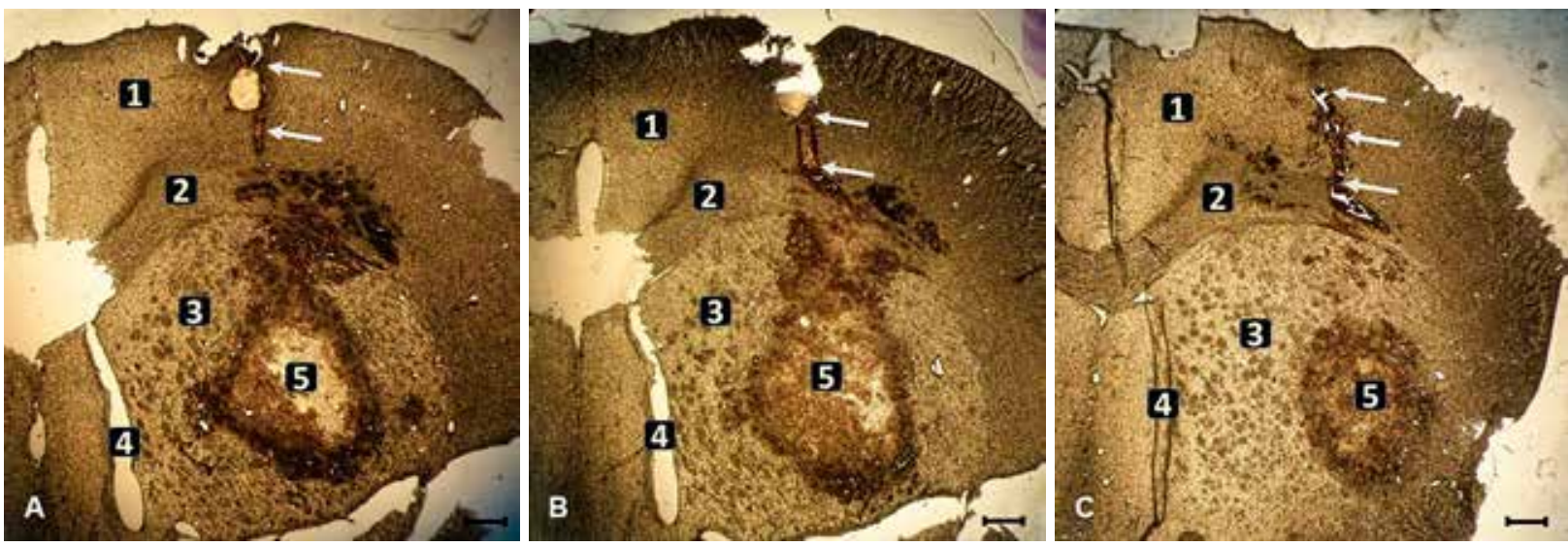

Fig. 1. Microphotographs of sections through ICH, where traces of needle insertion can be seen (marked by arrows) 1 cerebral cortex; 2 - corpus callosum; 3 - striatum, 4 - lateral ventricle; 5 - ICH zone. Scale bar: $500 \mu \mathrm{m}$.

Table 3. Localization of actual collagenase injection places and the central part of ICH according to the stereotactic coordinates from bregma

\begin{tabular}{|l|r|r|r|r|r|r|r|r|}
\hline & $\mathbf{n}$ & $\mathbf{m i n}-\mathbf{m a x}$ & $\mathbf{Q}_{\mathbf{2 5}}$ & $\mathbf{Q}_{\mathbf{5 0}} \mathbf{( M e )}$ & $\mathbf{Q}_{\mathbf{7 5}}$ & \multicolumn{1}{c|}{$\mathbf{S k}$} & \multicolumn{1}{c|}{$\mathbf{K}$} & \multicolumn{1}{c|}{ AM } \\
\hline Actual place of injection (ML), mm & 7 & $2.6-3.3$ & 3.0 & 3.0 & 3.2 & -0.7 & 1.3 & 3.0 \\
\hline Actual place of injection (AP), mm & 7 & $0.6-1.8$ & 1.0 & 1.2 & 1.5 & -0.3 & -0.6 & 1.2 \\
\hline Central part of the ICH (AP), mm & 7 & $0.4-1.4$ & 0.5 & 0.6 & 0.8 & 1.7 & 3.3 & 0.7 \\
\hline $\begin{array}{l}\text { The displacement of the ICH center from the } \\
\text { preplanned place of needle insertion (AP), mm }\end{array}$ & 7 & $0.2-1.2$ & 0.3 & 0.4 & 0.6 & 1.7 & 3.3 & 0.5 \\
\hline $\begin{array}{l}\text { The displacement of the ICH center from the } \\
\text { actual place of needle insertion (AP), mm }\end{array}$ & 7 & $-0.2--1$ & -0.3 & -0.5 & -0.7 & -0.4 & -1.0 & -0.5 \\
\hline
\end{tabular}

Where: min-max is the minimum and maximum value, $\mathrm{Q}_{25}, \mathrm{Q}_{50}$ and $\mathrm{Q}_{75}$ are the $1 \mathrm{st}$, $2 \mathrm{nd}$, and 3rd quartiles respectively; Me is the median; Sk - skewness; $\mathrm{K}$ - kurtosis; AM - arithmetic mean; ICH - intracerebral hemorrhage.

Table 4. Morphological characteristics of the ICH sections

\begin{tabular}{|l|l|l|l|l|l|l|l|l|l|l|l|}
\hline & $\mathbf{n}$ & $\mathbf{m i n}$ & $\mathbf{Q}_{\mathbf{2 5}}$ & $\mathbf{Q}_{\mathbf{5 0}} \mathbf{( M e )}$ & \multicolumn{1}{|c|}{$\mathbf{Q}_{\mathbf{7 5}}$} & $\mathbf{m a x}$ & $\mathbf{~ S k}$ & $\mathbf{K}$ & $\mathbf{A M}$ & $\mathbf{S D}$ & $\mathbf{M}$ \\
\hline Shape factor (SF) & 131 & 0.09 & 0.20 & 0.32 & 0.50 & 0.86 & 0.61 & -0.62 & 0.36 & 0.19 & 0.02 \\
\hline The depth of MZ, mm & 78 & 0.40 & 0.51 & 0.56 & 0.59 & 0.65 & -0.49 & -0.41 & 0.55 & 0.07 & 0.02 \\
\hline $\begin{array}{l}\text { The ICH area, mm } \text { mm }^{2} \\
\text { (sections without CZ) }\end{array}$ & 53 & 0.1 & 0.8 & 1.5 & 2.3 & 9.7 & 2.6 & 8.9 & 1.85 & - & - \\
\hline $\begin{array}{l}\text { The ICH area, mm }{ }^{2} \\
\text { (sections with CZ) }\end{array}$ & 78 & 1.3 & 4.2 & 6.1 & 8.8 & 11.5 & 0.05 & -1.1 & 6.47 & 2.88 & 0.33 \\
\hline
\end{tabular}

Notes: min-max is the minimum and maximum value, $\mathrm{Q}_{25}, \mathrm{Q}_{50}$ and $\mathrm{Q}_{75}$ are the 1 st, 2 nd, and 3rd quartiles respectively; Me is the median; Sk - skewness; $\mathrm{K}$ - kurtosis; AM - arithmetic mean; SD - standard deviation; $\mathrm{M}$ - error of the mean; ICH - intracerebral hemorrhage; MZ - marginal zone; CZ - clear zone (zone of enlightenment). 

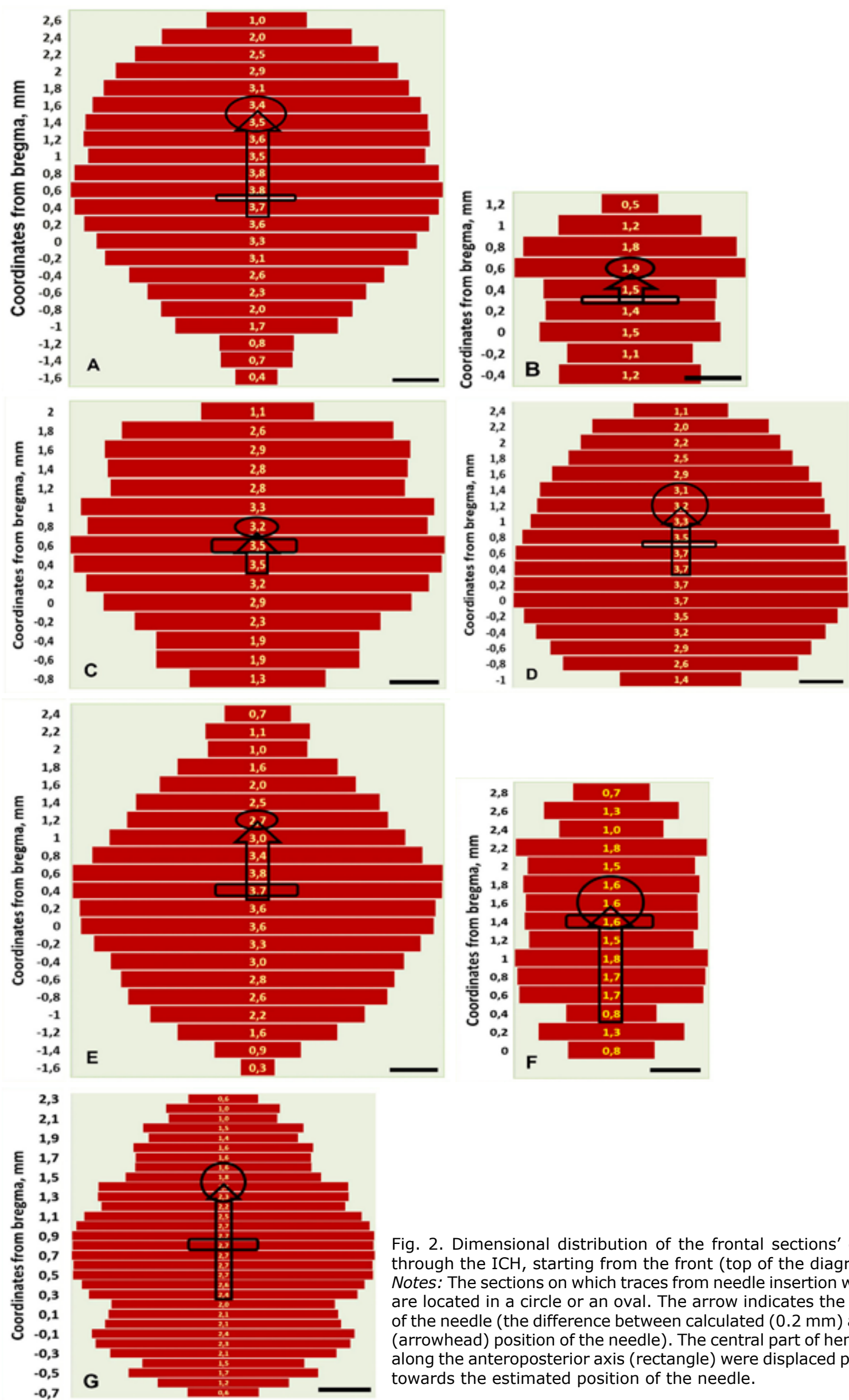

Fig. 2. Dimensional distribution of the frontal sections' diameters through the $\mathrm{ICH}$, starting from the front (top of the diagram). Notes: The sections on which traces from needle insertion were found are located in a circle or an oval. The arrow indicates the deflection of the needle (the difference between calculated $(0.2 \mathrm{~mm})$ and actual (arrowhead) position of the needle). The central part of hemorrhages along the anteroposterior axis (rectangle) were displaced posteriorly, towards the estimated position of the needle. 

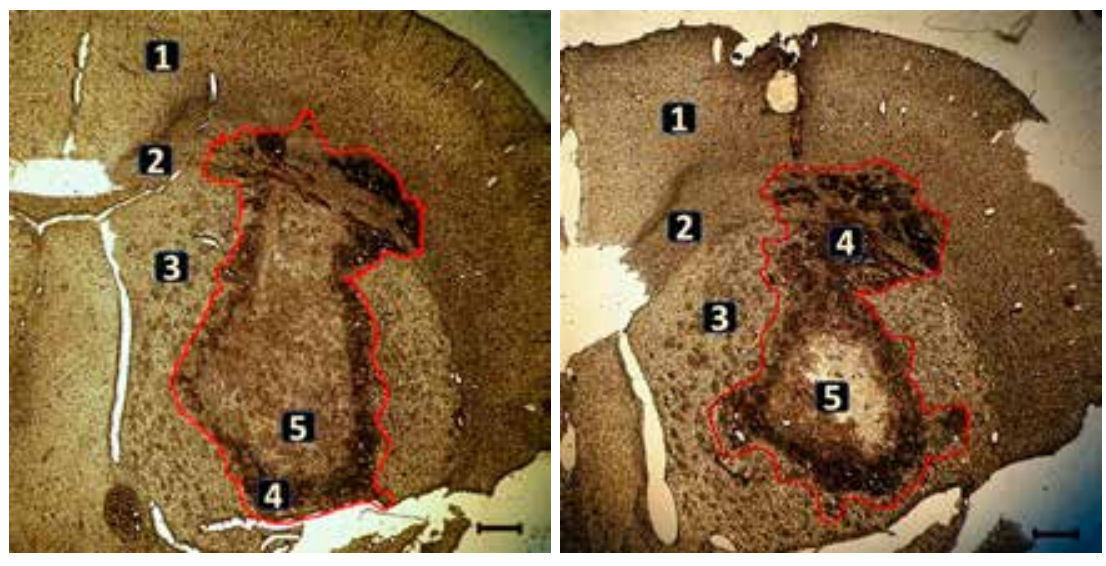

Fig. 3. Microphotographs of intracerebral hemorrhage induced by the collagenase injection into the rat striatum. The boundaries of $\mathrm{ICH}$ are highlighted in red. 1 - cerebral cortex; 2 - corpus callosum; 3 striatum, 4 - marginal zone; 5 zone of enlightenment. Scale bar: $500 \mu \mathrm{m}$

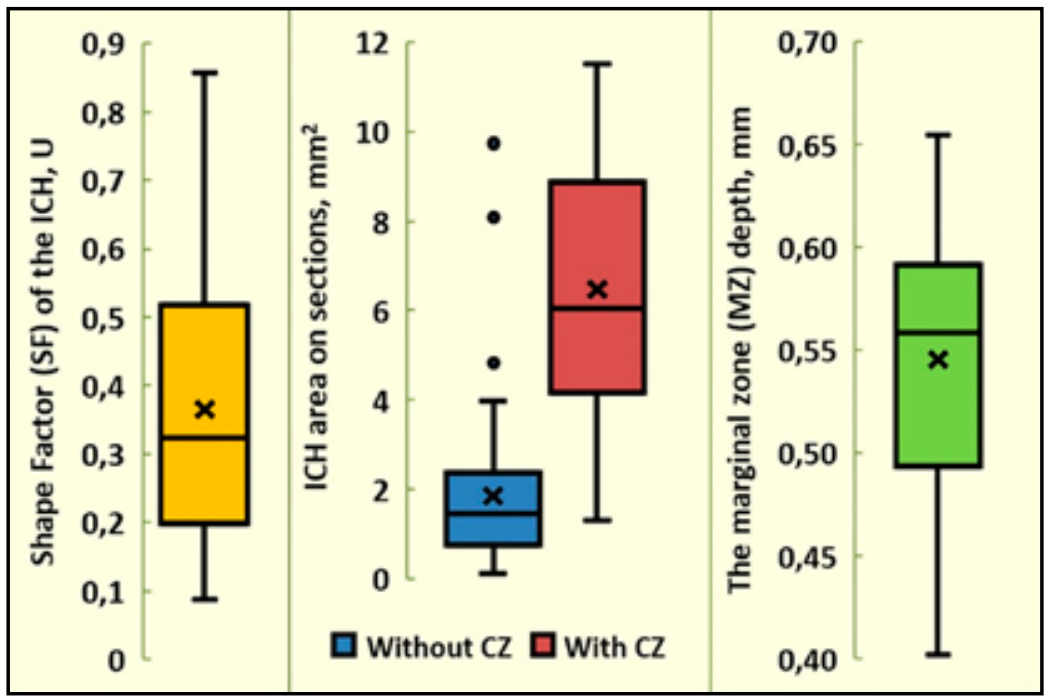

Fig. 4. Morphological features of marginal and central zones of the hemorrhage. Notes: Box and whiskers plot indicates characteristics of distributions: the line in the middle is the median (2nd quartile); the lower and upper borders of boxes are the 1st and 3rd quartiles, respectively; The whiskers are the minimum and maximum values; " $X$ " is the mean.

The study of the animals' motion demonstrated that each of the tests had a limited prognostic value and described individual aspects of nervous system disorders in the animals (Fig. 5). The BWt made it possible to measure hindlimb functioning in rats and evaluate their coordination of movements, so it turned out to be a valuable addition to the other tests. However, when studying the dependence of test results on the volume of hemorrhage, a non-linear quadratic correlation was found ( $\mathrm{R}=0.93 ; p=0.02$ ) (Fig. 5A), so this test, by itself, cannot be used to predict hemorrhage volume. (Fig. $3 \boldsymbol{A}$ ) This probably can be explained by the change in rats' dominant emotions which depend on the severity of ICH. Even in the mildest form of ICH the rats seem to show unwillingness to cross the beam due to weakness and apathy. In a moderate form, rats cross the beam because of fear to fall off. In the most severe form, animals are not able to move and, remain on the beam despite of fear of falling.

In ARt and NSt linear correlations were observed between the results of these tests and the amount of hemorrhage although not reliable $(R=0,66$ and 0,67 respectively; $p=0.103$ and 0.100 respectively) (Fig. $5 \mathrm{~B}, \boldsymbol{C})$.

To use more balanced and comprehensive assessment of the neurological status of the rats in predicting the amount of hemorrhage, it would be advisable to apply an integrated calculated index that includes the results of each of the tests performed. Since all the tests had a point score, we can sum the test scores, where their number is directly proportional to the severity of animals impairment (BWt and ARt) and subtract the points obtained in that test which results are inversely proportional to the impairment in the rats (NSt). Thus, an integrated neurological estimation will be the result of summing BWt and ARt scores and subtracting NSt scores from them. The tests used for calculation have the following ranges of possible values: BWt - from 0 to 6 points, ARt - from 0 to 4 points, and NSt - from 0 to 8 points. The total range of possible values corresponds to: minimum value $=0+0-8$ $=-8$ points; maximum value $=6+4-0=10$ points. As a result, the assessment on an integrated scale can have 19 possible gradations (from 0 to 18 ), at the same time there are negative values in it, which is not convenient for displaying the results. Negative numbers can be eliminated by adding a constant ' 8 ' to the formula:

$I N E=B W t+A R t-N S t+8(4)$

where INE is an integrated neurological estimation, BWt is a beam walking test; ARt - adhesive removal test; NSt - neurological score test; 8 is a constant.

Thus, the formula will have 19 possible points from 0 to 18 , where all possible values will be positive.

The study of the correlation between INE and hemorrhage amount (Fig. $3 \mathrm{D}$ ) has confirmed the greater 

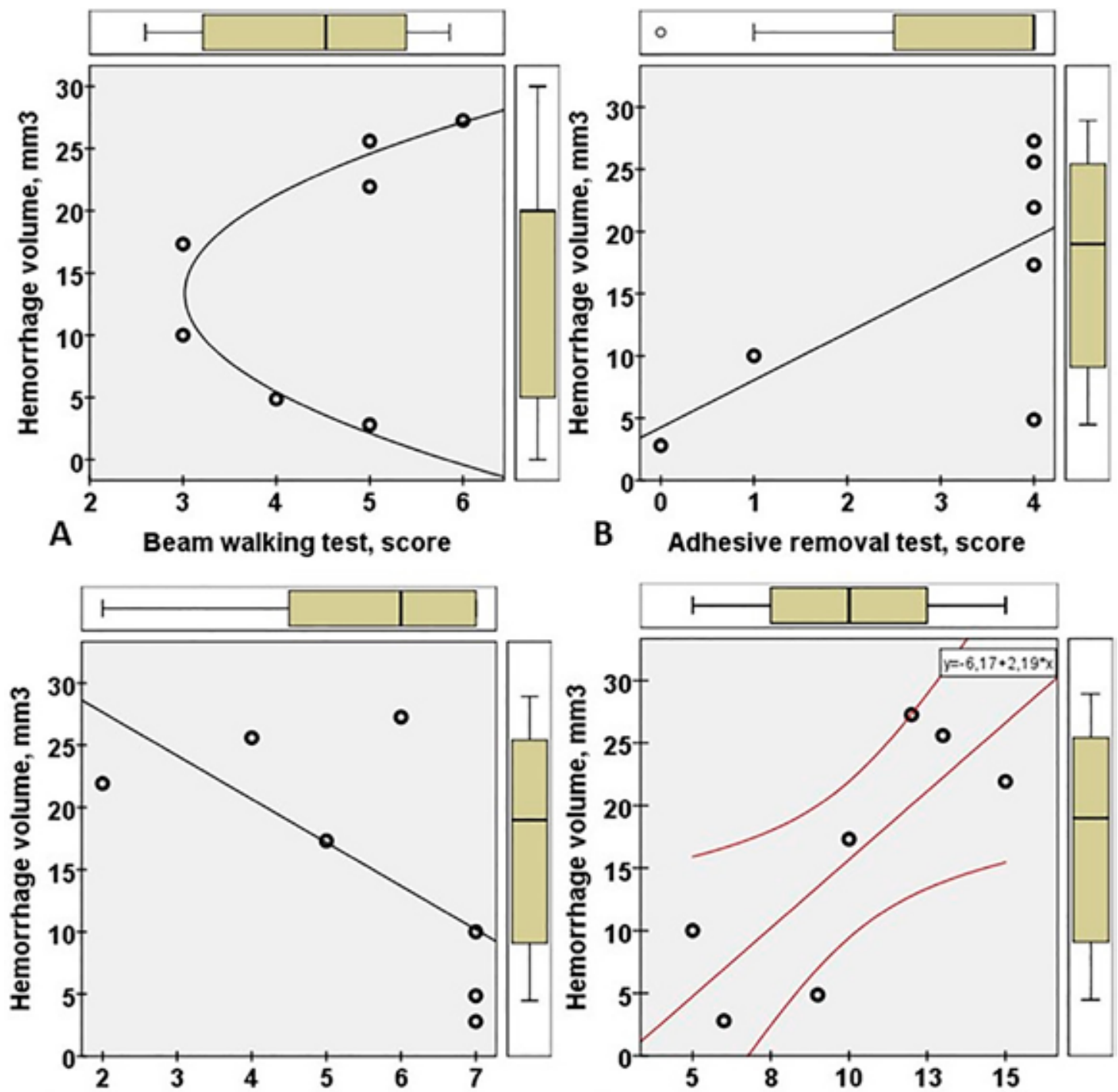

C
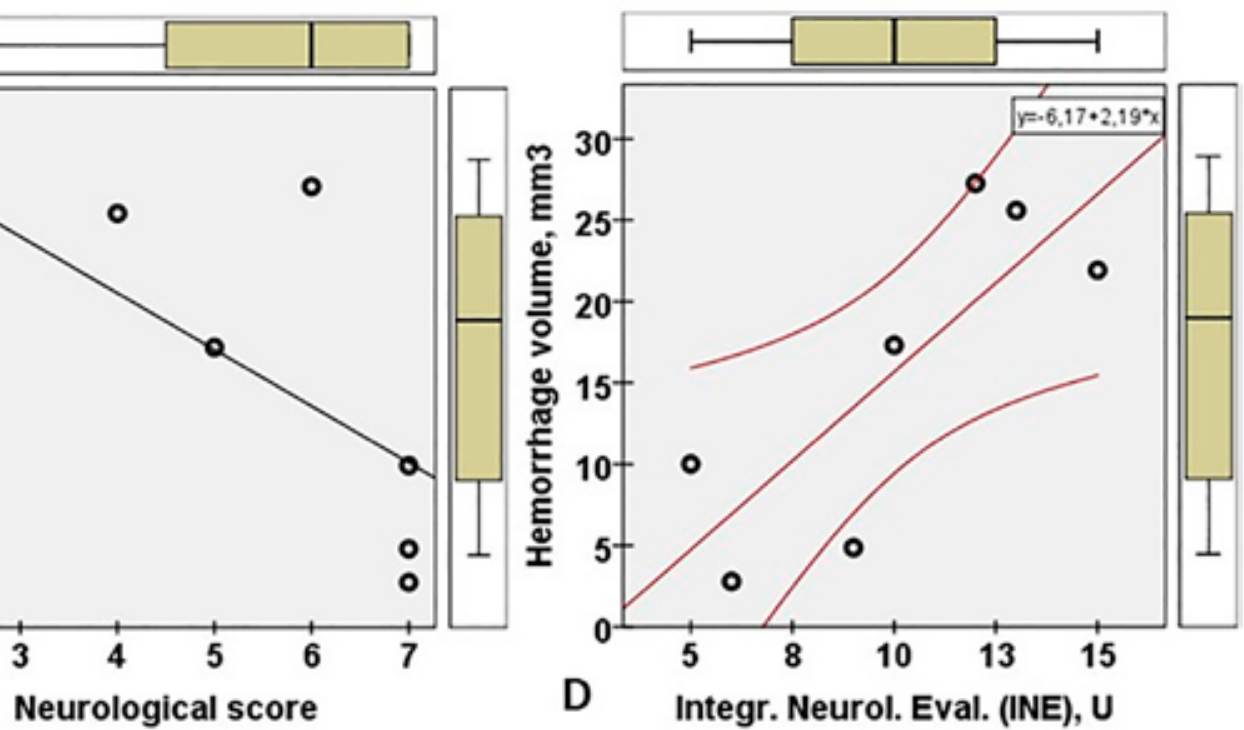

Fig. 5. Diagrams of dependence of behavioral tests results on hemorrhage volume (linear and curvilinear corellatios). Notes: Box and whiskers plot indicates characteristics of distributions: the line in the middle is the median (2nd quartile); the lower and upper borders of boxes are the 1st and 3rd quartiles, respectively; The whiskers are the minimum and maximum values; " $\mathrm{X}$ " is the mean.

prognostic value of INE for studying hemorrhage than each of the tests individually. As a result, a statistically significant positive correlation was obtained $(\mathrm{R}=0,806 ; p=0.029)$, on which base a linear regression formula was maid:

ICH volume $=2.19 \times I N E-6.17 ;(5)$

hence:

$I C H$ volume $=2.19 \times(B W t+A R t-N S t+8)-6.17 ;(6)$

$\mathrm{ICH}$ volume $=2.19 \times(B W t+A R t-N S t)+11.35(7)$

where 6.17 - regression constant; 2.19 - regression coefficient B; BWt - Beam Walking Test; ARt - Adhesive Removal Test; NSt - Neurological Score Test.

Using obtained regression formula (7) allows you to study the amount of hemorrhage in rats more accurately on the 1st day after ICH modeling in the absence of MRI results. INE, unlike each of the included tests, has a normal distribution. This may reflect a lesser exposure of this indicator to the influence of random factors related to variability in animal behavior.
It should be noted that INE also has independent value, since the correct assessment of the dysfunction severity may be of greater importance than any instrumental and laboratory methods.

The obtained results indicate a significant range of variation in ICH volume in the collagenase model (from 3 to $27 \mathrm{~cm}^{3}$ ) (Table 2). Approximately one third of the animals showed a mild degree of dysfunction and a small amount of hemorrhage. The remaining rats had moderate $\mathrm{ICH}$ severity (Fig. 5). When compared the range of $\mathrm{ICH}$ volume variation with other research groups [4, 5, 14-17], the modeling of ICH was carried out by injection of 0.14 to 0.4 units of bacterial collagenase, minimum and maximum values were not shown, nevertheless the arithmetic means and standard deviations (SD) were indicated, hence coefficients of variation (CV), which formed 6\% [5], 16\% [14] 21\% [15] $33 \%$ [4], 40\% [16], and 52\% [17] were figured out. In 
our study, CV was $63 \%$, which is higher than in other publications. In our opinion, this can be explained by the genetic heterogeneity of animals (our study was carried out on outbred rats), the rejection of rats with insufficient hemorrhage volume by other authors when the main study was not dedicated to the model investigation, but to another topic, as well as random events or other unpublished circumstances of the experiments.

In most cases, the shape of the hemorrhage was irregular and elongated, mainly posterior to the area of collagenase injection. Elongation in the dorsoventral direction near the injection site was also noted, which repeated the way the needle was inserted during $\mathrm{ICH}$ modeling (Table 3, Fig. 1; 3). The edges of the ICH 0.56 $(0.5 ; 0.6) \mathrm{mm}$ depth were characterized by more intense hemosiderin staining than the central part. It indicated multiple repeated diapedetic hemorrhages during the day after the ICH modeling, which was alternated with the periods of hemostasis. This should have led to a longer dysfunction of the blood-brain barrier than in the model where the whole blood was injected.

Since the collagenase model is associated with a significant variability in both the amount of hemorrhage and the severity of functional disorders in animals, it seems necessary to select the rats with a sufficient degree of dysfunction in behavioral tests and a certain amount of hemorrhage. The obtained INE index allows us not only to select animals correctly for the experiment, but also to calculate the probable volume of hemorrhage on the 1st day after the collagenase injection using the regression model.

\section{Conclusions}

1. The collagenase model of intracerebral hemorrhage is associated with a large variability of dysfunction in rats, as well as the volume of hemorrhage (from 3 to $27 \mathrm{~cm}^{3}$ ).

2. ICH had an irregular rugged shape and marks of repeated diapedetic hemorrhages of about $0.6 \mathrm{~mm}$ depth.

3. The center of ICH along the anteroposterior axis was on average $0.5 \mathrm{~mm}$ posterior to the actual site of collagenase injection.

4. The combined use of the neurological score test, the beam walking test and the adhesive removal test in the collagenase ICH model makes it possible not only to determine the degree of functional disorder in the animals more accurately, but also to estimate the probable amount of intracerebral hemorrhage on the 1st day by using the regression method.

\section{Disclosure}

\section{Conflict of interest}

The authors declare no conflict of interest.

Ethical approval

All procedures performed on experimental animals comply with the regulations and ethics and approved by the Ethics and Bioethics Commission of Institute for Problems of Cryobiology and Cryomedicine of the NAS of Ukraine (Meeting Minutes No 5 of 08 December 2020).

\section{Funding}

The research had no sponsor support.

\section{References}

1. An SJ, Kim TJ, Yoon BW. Epidemiology, Risk Factors, and Clinical Features of Intracerebral Hemorrhage: An Update. J Stroke. 2017 Jan;19(1):3-10. doi: 10.5853/jos.2016.00864.

2. Godoy DA, Núñez-Patiño RA, Zorrilla-Vaca A, Ziai WC, Hemphill JC. Intracranial Hypertension After Spontaneous Intracerebral Hemorrhage: A Systematic Review and Metaanalysis of Prevalence and Mortality Rate. Neurocrit Care. 2019 Aug;31(1):176-87. doi: 10.1007/s12028-018-0658-x.

3. Fiorella D, Zuckerman SL, Khan IS, Ganesh Kumar N, Mocco J. Intracerebral Hemorrhage: A Common and Devastating Disease in Need of Better Treatment. World Neurosurg. 2015 Oct;84(4):1136-41. doi: 10.1016/j.wneu.2015.05.063.

4. MacLellan $C L$, Silasi $G$, Poon $C C$, Edmundson $C L$, Buist $R$, Peeling J, Colbourne F. Intracerebral hemorrhage models in rat: comparing collagenase to blood infusion. J Cereb Blood Flow Metab. 2008 Mar;28(3):516-25. doi: 10.1038/ sj.jcbfm.9600548.

5. Sang YH, Liang YX, Liu LG, Ellis-Behnke RG, Wu WT, So $\mathrm{KF}$, Cheung RT. Rat model of intracerebral hemorrhage permitting hematoma aspiration plus intralesional injection. Exp Anim. 2013;62(1):63-9. doi: 10.1538/expanim.62.63.

6. Paxions G, Watson C. The rat brain in stereotaxic coordinates: Compact. 7th ed. Sydney: Academic Press; 2017. 388p.

7. Beray-Berthat V, Delifer C, Besson VC, Girgis H, Coqueran B, Plotkine M, Marchand-Leroux C, Margaill I. Longterm histological and behavioural characterisation of a collagenase-induced model of intracerebral haemorrhage in rats. J Neurosci Methods. 2010 Aug 30;191(2):180-90. doi: 10.1016/j.jneumeth.2010.06.025.

8. Urakawa S, Hida H, Masuda T, Misumi S, Kim TS, Nishino H. Environmental enrichment brings a beneficial effect on beam walking and enhances the migration of doublecortin-positive cells following striatal lesions in rats. Neuroscience. 2007 Feb 9;144(3):920-33. doi: 10.1016/j.neuroscience.2006.10.038

9. Ross MH. Histology: a text and atlas with correlated cell and molecular biology. 7th ed. 2016. 984 p.

10. Kvitnitskaia-Ryzhkova TYu, Bozhok YuM, editors. Introduction to quantitative histology. Kiev: Kniga-plus; 2013. 256 p. Russian.

11. Artamentova LO, Utevska OM. Statistics for biologists. Kharkiv: HTMT; 2014. 331 p. Ukrainian.

12. McDonald JH. Handbook of biological statistics. Baltimore: Sparky House Publishing; 2014. 299 p.

13. Perry LA, Rodrigues M, Al-Shahi Salman R, Samarasekera $\mathrm{N}$. Incident cerebral microbleeds after intracerebral hemorrhage. Stroke. 2019 Aug;50(8):2227-2230. doi: 10.1161/STROKEAHA.118.023746.

14. Tao Y, Tang J, Chen Q, Guo J, Li L, Yang L, Feng H, Zhu G, Chen Z. Cannabinoid CB2 receptor stimulation attenuates brain edema and neurological deficits in a germinal matrix hemorrhage rat model. Brain Res. 2015 Mar 30;1602:127-35. doi: $10.1016 /$ j.brainres.2015.01.025

15. Kitaoka T, Hua Y, Xi G, Hoff JT, Keep RF. Delayed argatroban treatment reduces edema in a rat model of intracerebral hemorrhage. Stroke. 2002 Dec;33(12):3012-8. doi: 10.1161/01.str.0000037673.17260.1b.

16. Klahr AC, Dietrich K, Dickson CT, Colbourne F. Prolonged Localized Mild Hypothermia Does Not Affect Seizure Activity After Intracerebral Hemorrhage in Rats. Ther Hypothermia Temp Manag. 2016 Mar;6(1):40-7. doi: 10.1089/ ther.2015.0028.

17. Klahr AC, Dickson CT, Colbourne F. Seizure activity occurs in the collagenase but not the blood infusion model of striatal hemorrhagic stroke in rats. Transl Stroke Res. 2015 Feb;6(1):29-38. doi: 10.1007/s12975-014-0361-y.2 